\title{
Specialised Image Capture Systems for a DIET Breast Cancer Screening System
}

3rd ASME/IEEE International Conference on Mechatronic and Embedded Systems and Applications MESA07

C. E. Hann ${ }^{1}$, J. G. Chase' ${ }^{1}$, C. Berg' ${ }^{1}$, R. G. Brown ${ }^{1}$, R. B. Elliot ${ }^{1}$, X. Chen ${ }^{1}$

${ }^{1}$ Department of Mechanical Engineering, University of Canterbury, Christchurch, New Zealand 


\section{Introduction}

- Breast cancer has second highest mortality rate of all cancers for women worldwide. In New Zealand it is number one.

- Mammography is the standard for breast cancer screening

- discomfort to patients and health risk (exposure to radiation)

- interpretation of images is subjective $\rightarrow$ misdiagnosis, false positives

- Digital Image-based Elasto-Tomography (DIET) is an emerging low cost technology for non-invasive breast cancer screening

- digital imaging of actuated breast to determine tissue motion

- 3D internal tissue stiffness reconstruction (finite element method)

- Regions of high stiffness suggest cancer

- Requires up to $100 \mathrm{~Hz}$ image capture (5-10 cameras ideally)

- Problem: Cameras too large and expensive ( $\sim 15-20 \mathrm{k}$ US each, high resolution)

- Solution: "Off the shelf" CMOS imaging sensors combined with Stroboscope 


\section{DIET system overview}

- The DIET system is broken down into 4 fundamental steps:

(1) Actuation $\rightarrow$ (2) Image Capture $\rightarrow$ (3) Motion Tracking and measurement $\rightarrow$

(4) Tissue stiffness reconstruction

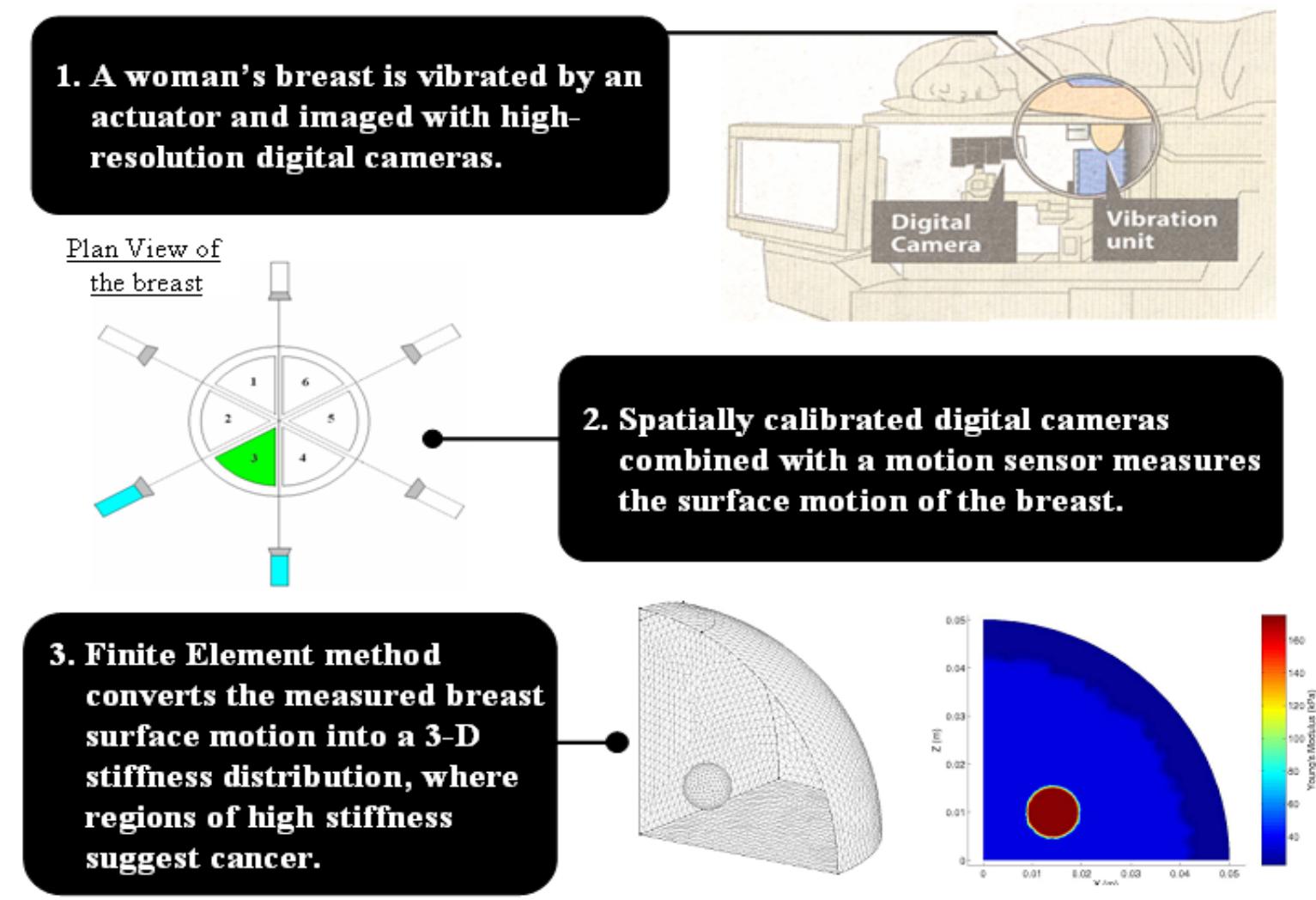




\section{Image capture}

- Image capture for DIET system $\rightarrow 50-100 \mathrm{~Hz}$

- This research implements stroboscope with Kodak's KAC-9648 color imaging (resolution of 1280x1024)

- Other high speed cameras are either expensive, bulky or have reduced resolution

- CMOS Sensors allow dense array of cameras placed about the breast

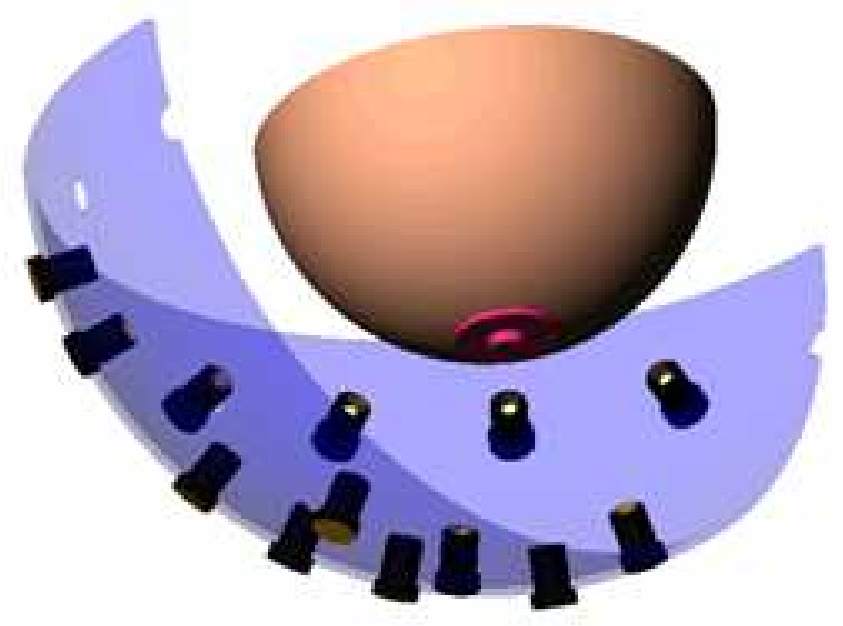




\section{Image capture apparatus and computer setup}
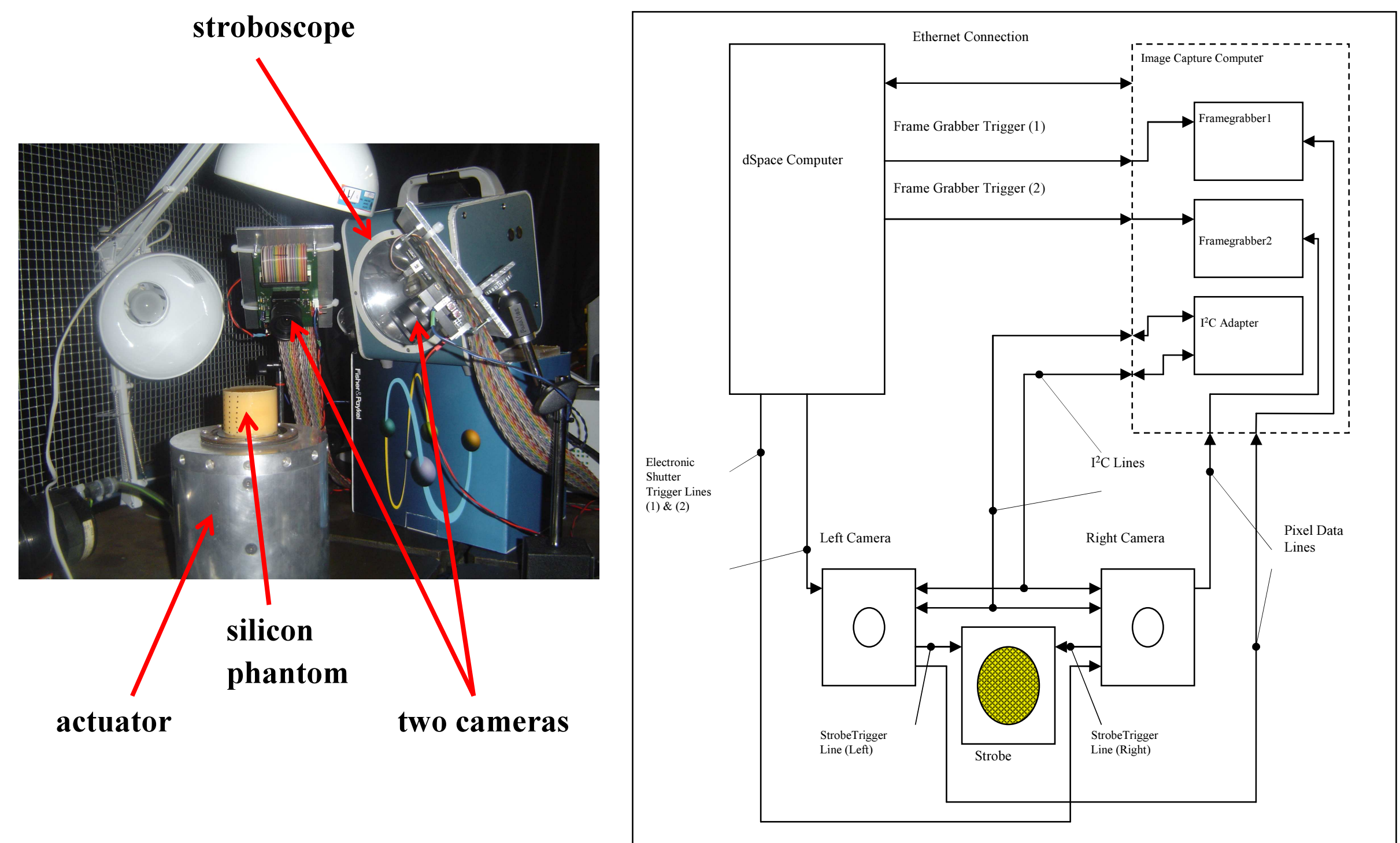


\section{Image capture - overview}

- The frame rate of each CMOS camera at $1280 \times 1024$ is approximately $18 \mathrm{fps}$

- To overcome this insufficient frame rate the breast is strobed at specific points in its motion

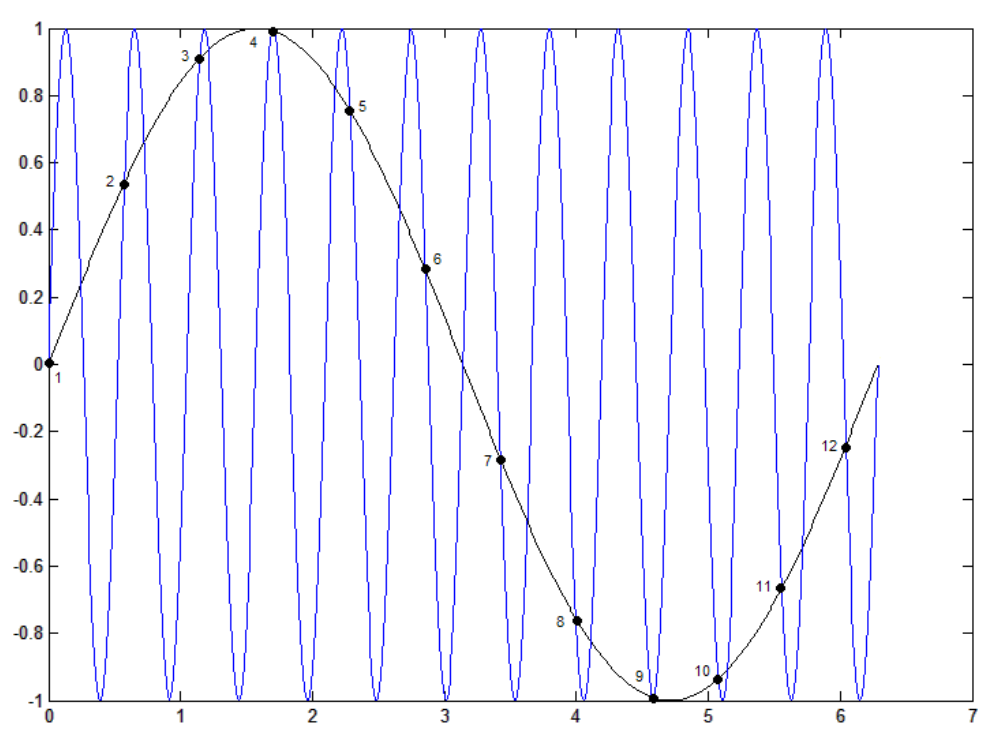

- In practice time between captured images 1-10 seconds $\rightarrow 100-1000$ cycles between images 


\section{The cameras}

- $\quad$ Two configurations for cameras, implemented using $\mathrm{I}^{2} \mathrm{C}$ bus:

(1) Initialization for camera $\rightarrow$ stream image data continuously to frame grabbers (to adjust colour gains, focus, camera position, aperture size)

(2) Allows camera to trigger strobe itself

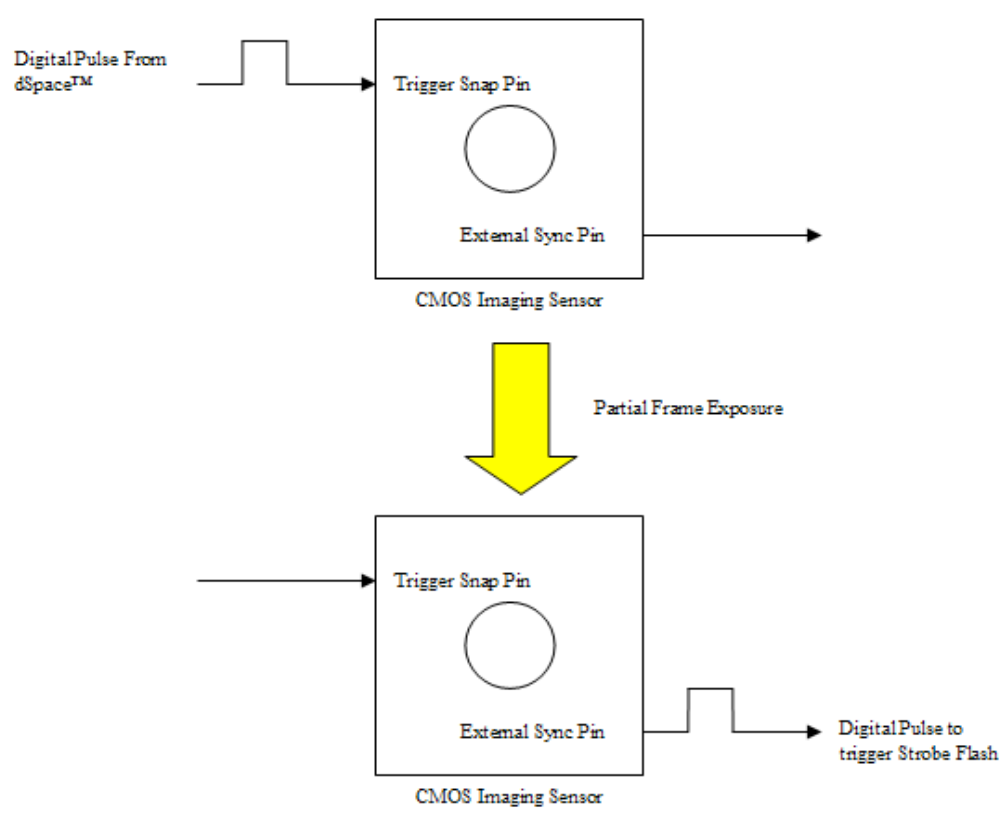

- $\quad$ First pin receives pulse and starts frame exposure

- $\quad$ Second pin supplies pulse to activate strobe 


\section{Actuator and Trigger Control}

- $\mathrm{dSpace} \mathrm{TM}^{\mathrm{TM}}$ control system drives image capture process (generates and synchronizes all signals)

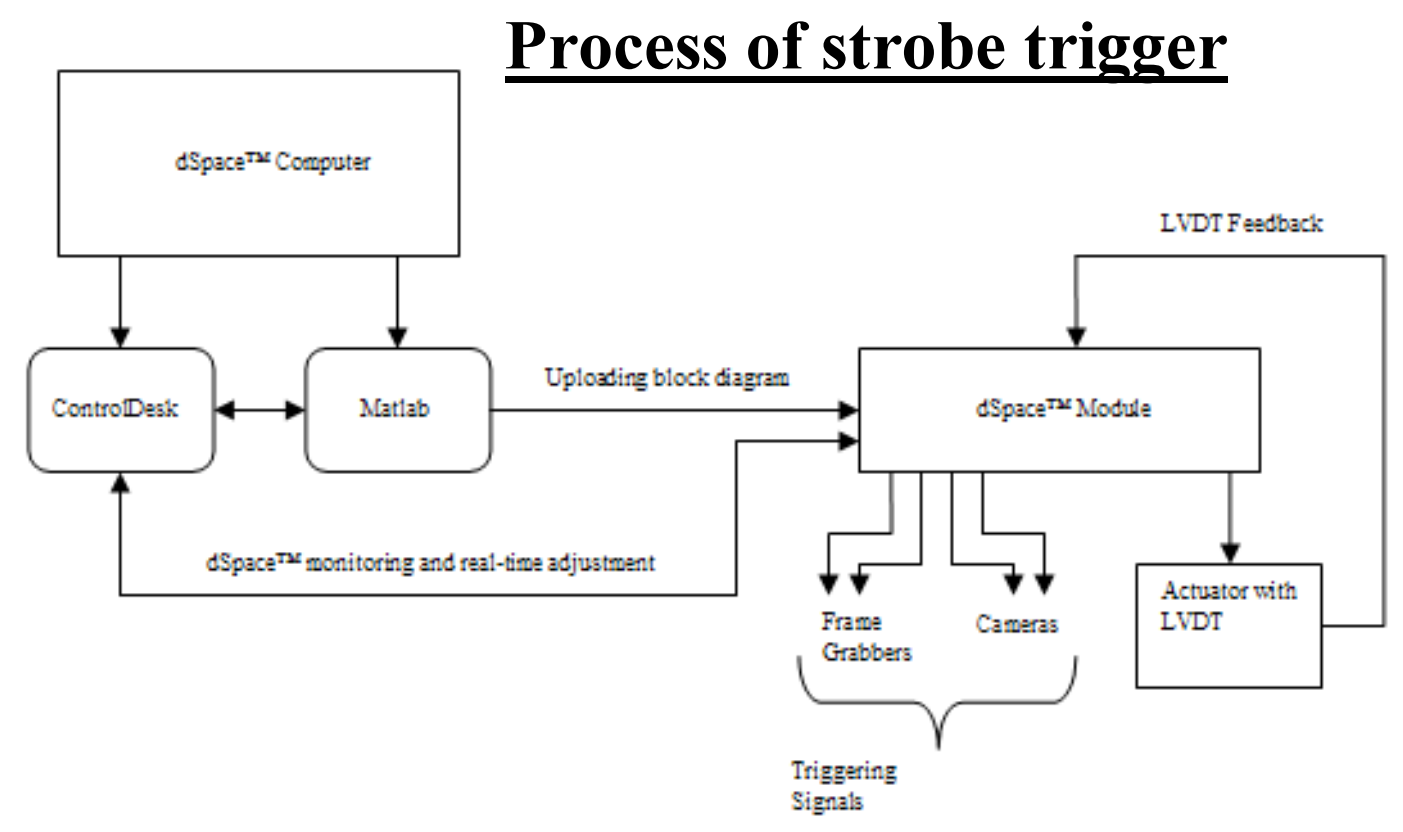

- $\quad$ SSpace ${ }^{\mathrm{TM}}$ drives trigger signals and actuator using loaded Simulink ${ }^{\mathrm{TM}}$ diagram

- ControlDesk ${ }^{\mathrm{TM}}$ software allows real-time adjustment of settings on dSpace ${ }^{\mathrm{TM}}$ module

- Python ${ }^{\mathrm{TM}}$ is used to automate ControlDesk ${ }^{\mathrm{TM}}$ 


\section{Image capture software - user interface}

- Features of the Image Capture Application

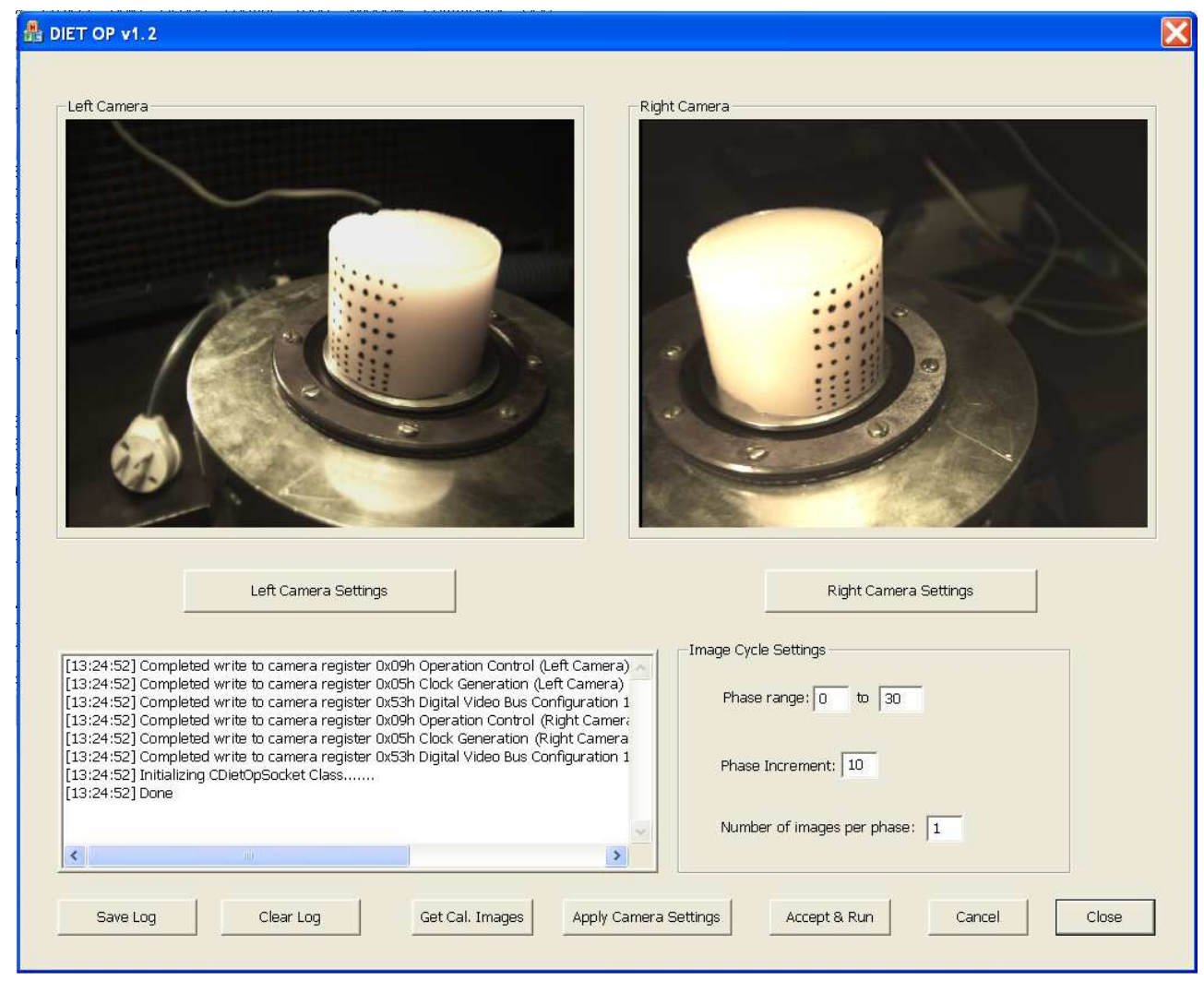

- Can look at images and make real time adjustments (camera position, colour, ...) 


\section{Preliminary results and problems}

- Time period between receiving trigger from dSpace ${ }^{\mathrm{TM}}$ and camera triggering strobe is inconsistent and unpredictable

- $\mathrm{dSpace}^{\mathrm{TM}}$ triggering is consistent

- Camera is consistent at a frequency of $10 \mathrm{~Hz}$ and not $100 \mathrm{~Hz}$
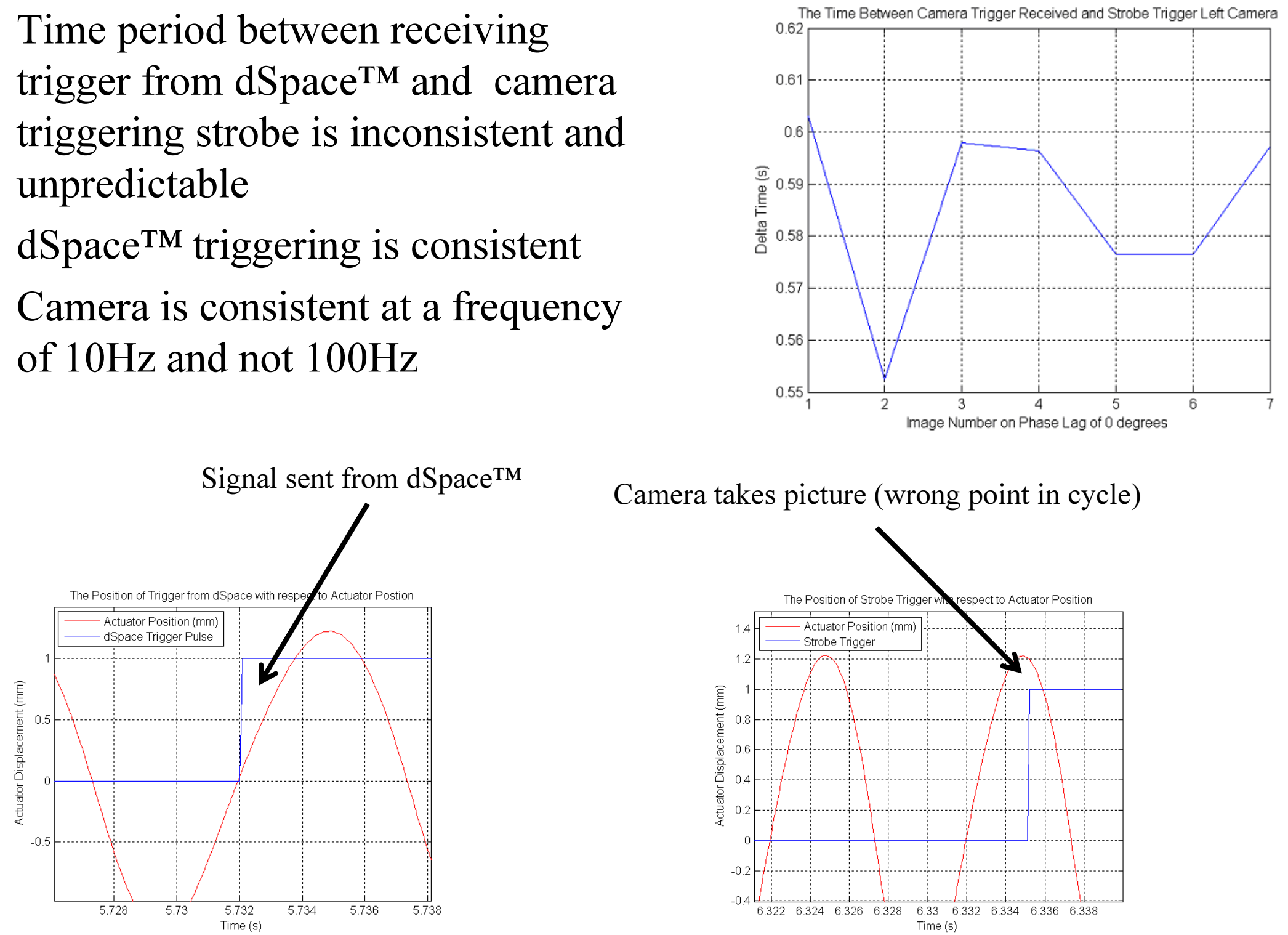

Camera takes picture (wrong point in cycle)

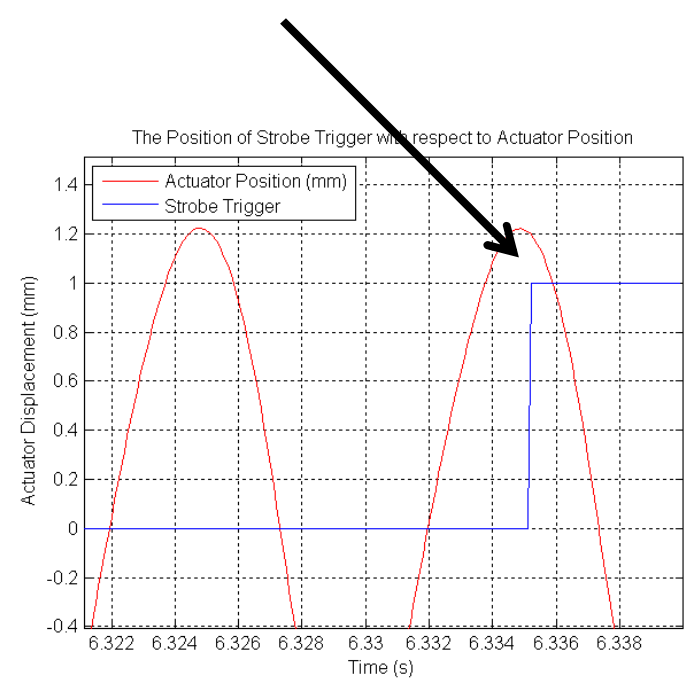




\section{Correcting the timing}

- The timing issues were solved by implementing an 'AND' gate and a feedback signal to dSpace ${ }^{\mathrm{TM}}$

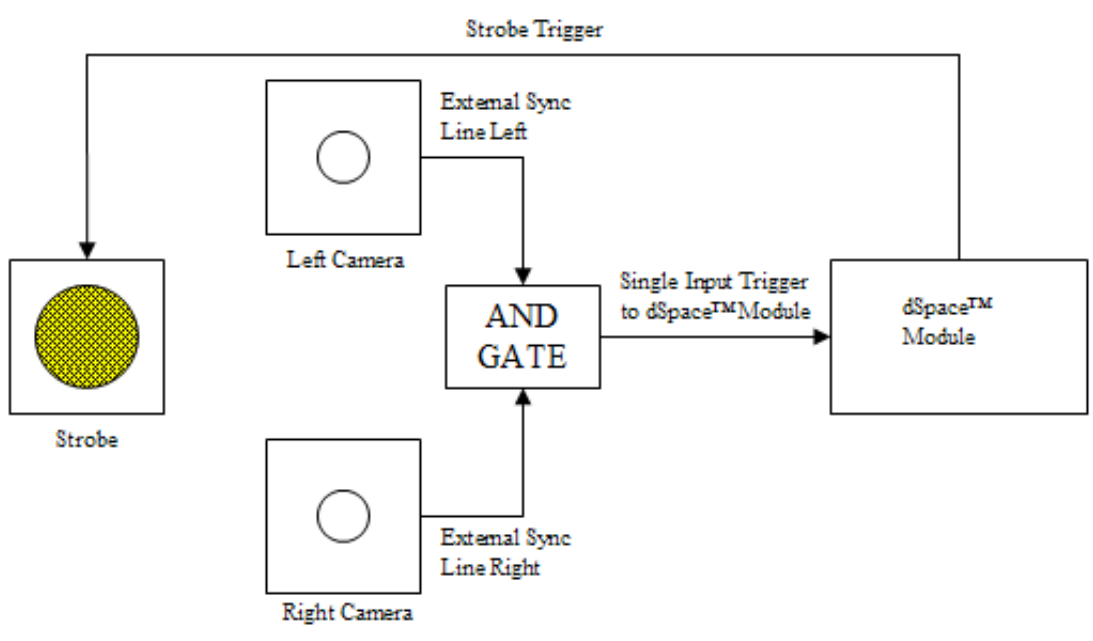

- When left AND right cameras are ready for strobe, $\mathrm{dSpace}{ }^{\mathrm{TM}}$ waits until the signal aligns with actuator, then it lets the strobe flash

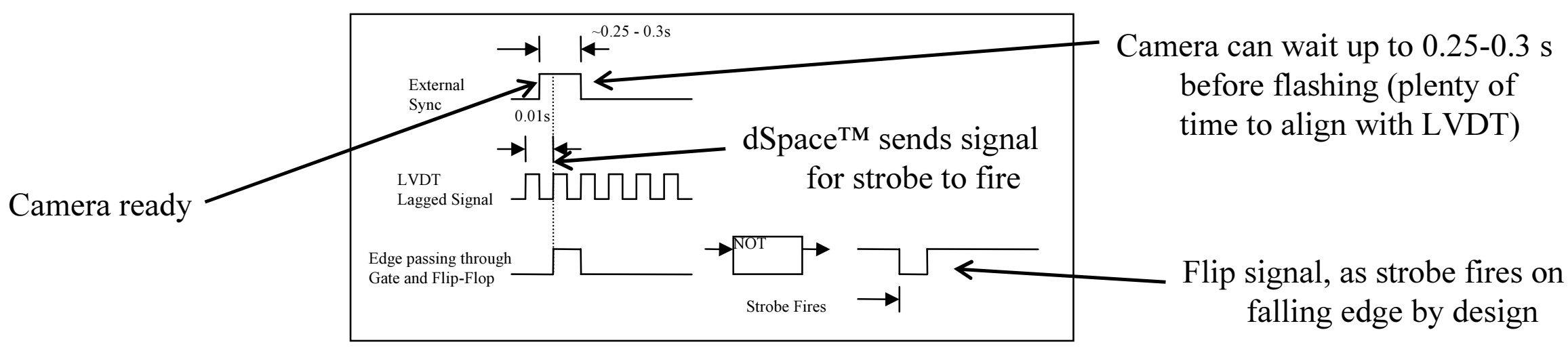




\section{Results - timing}

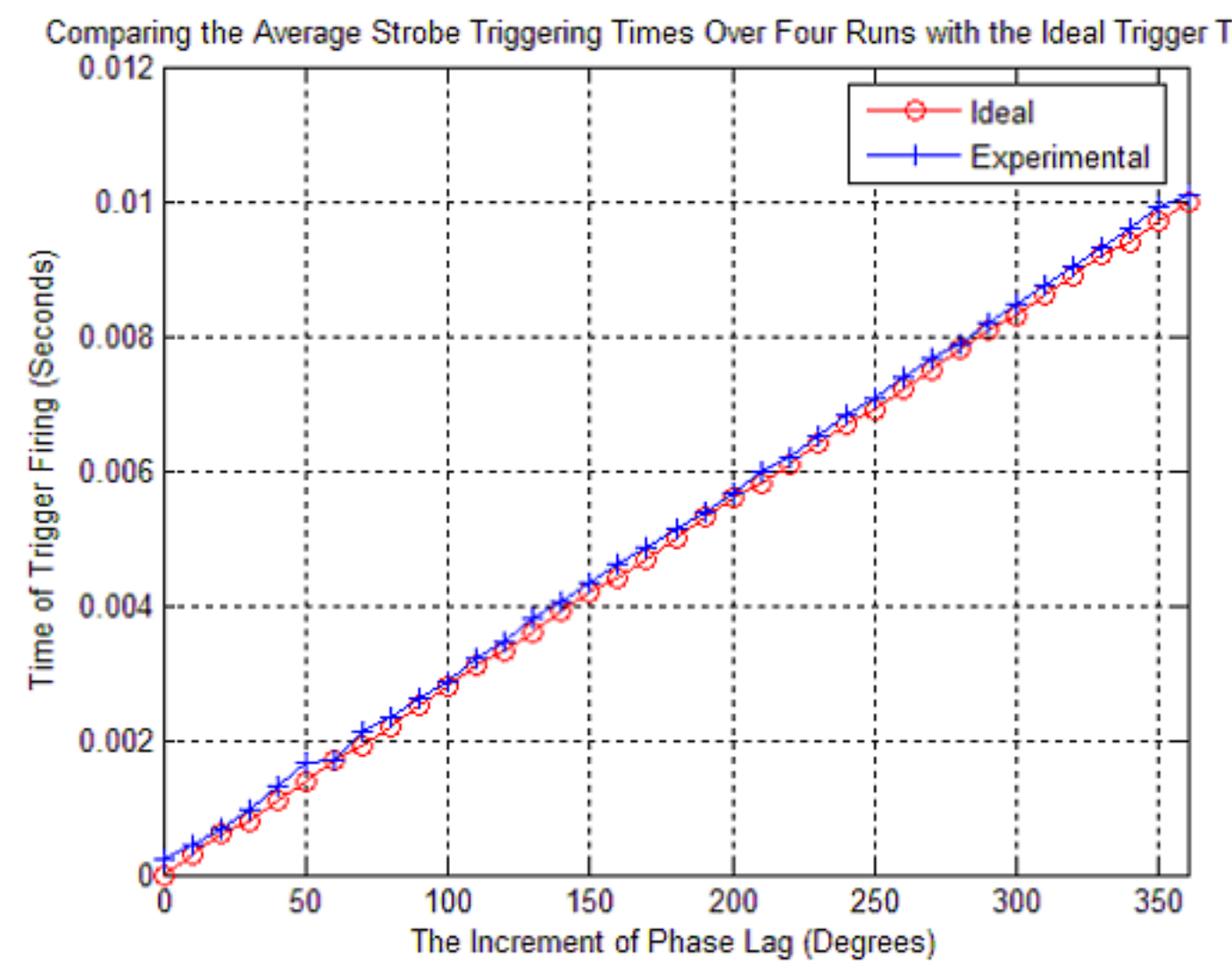

- Illustrates the accuracy in timing of the system

- Mean absolute error $\sim 1.4 \% \rightarrow$ time difference of 0.0002 seconds 


\section{Results - Actuator displacement vs ideal}

actuator displacements ( 10 degrees of phase) plotted as a waveform (total time $=\sim 6$ minutes)

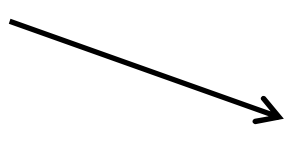

snap shot of actuators motion at $\mathrm{t}=1.82 \mathrm{~s}$

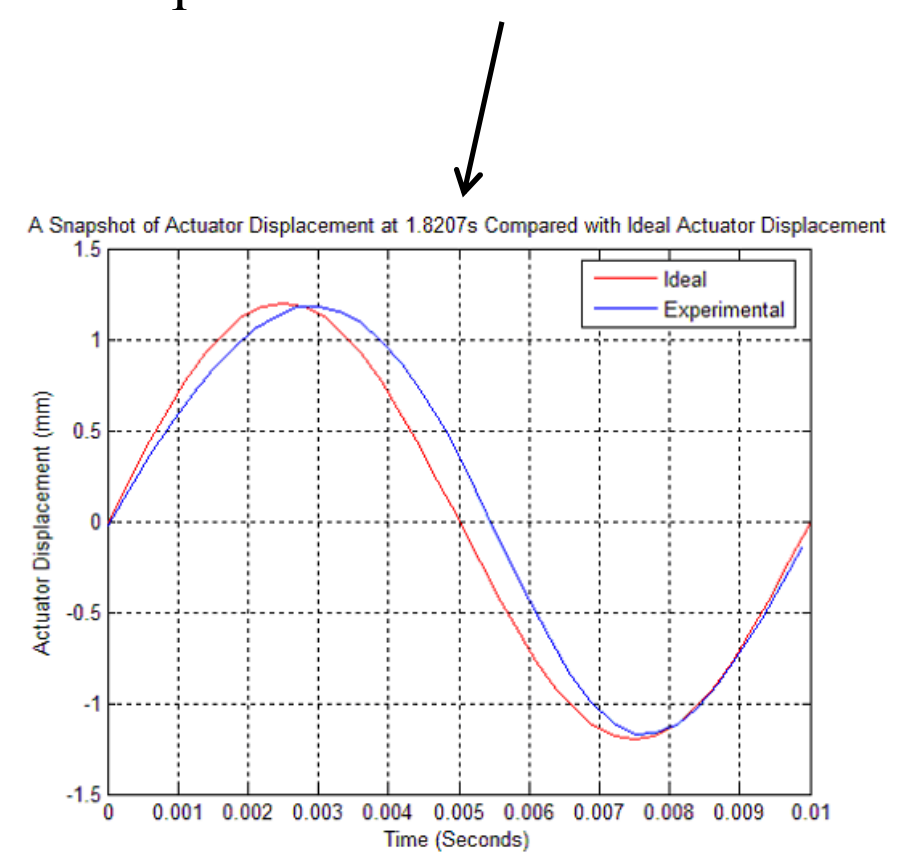

average of 20,000 waveforms (200 seconds)

- Results consistent $\rightarrow$ errors are physical limitations

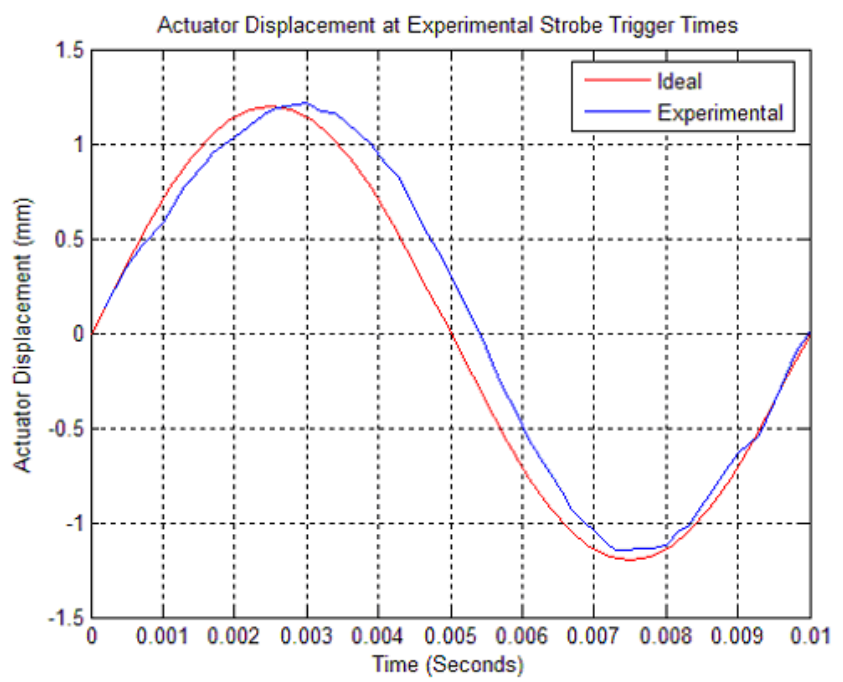
of current actuator (frequency varies from 95-100 Hz)

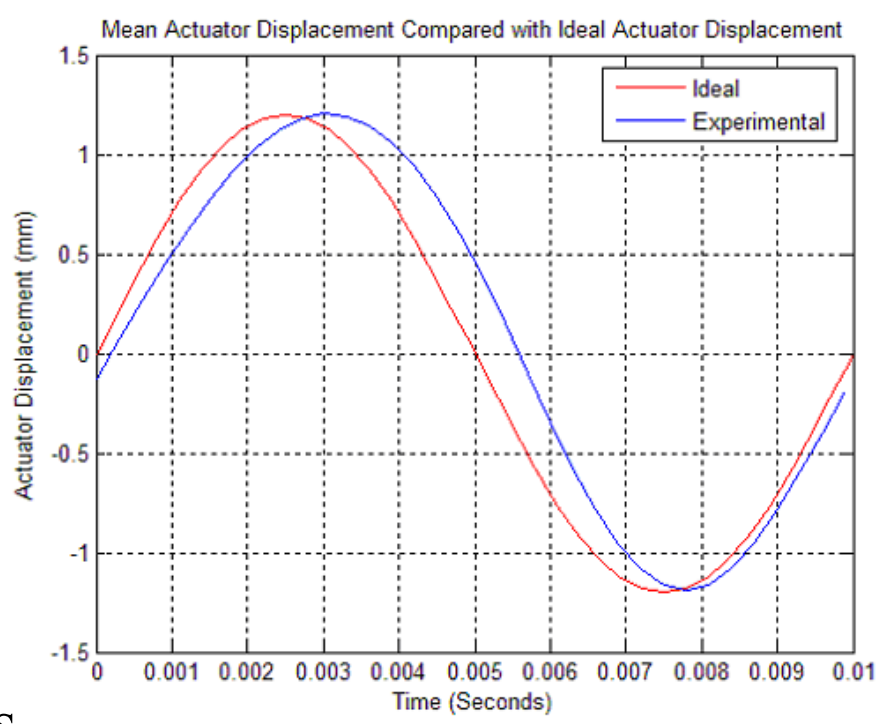




\section{Experimental results}

CANTERBURY

Te Whare Wänanga o Waitaha
CHRISTCHURCH NEW ZEALAND
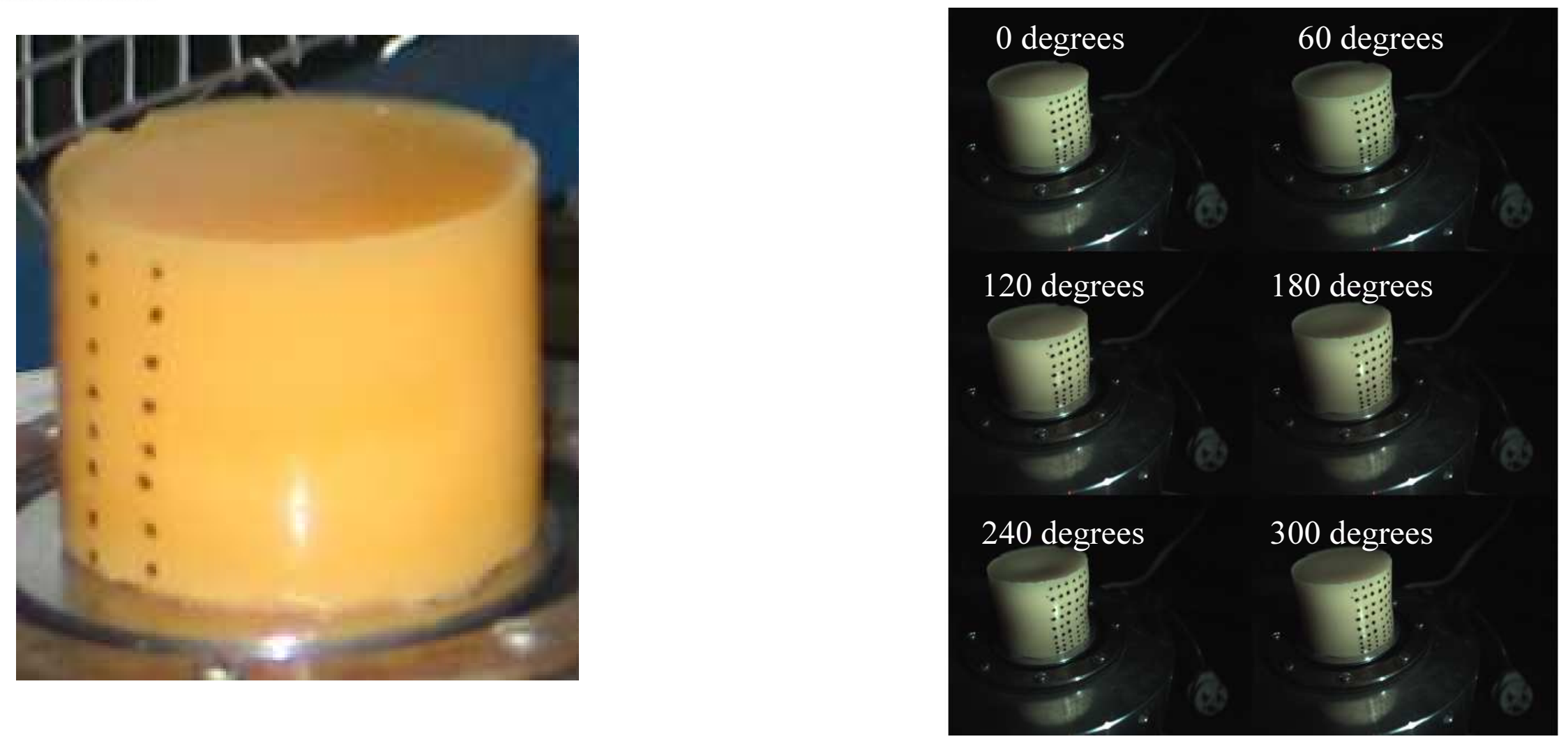

- $\quad$ Silicon phantom $50 \mathrm{~mm}$ diameter, $40 \mathrm{~mm}$ height

- $\quad$ Actuation frequency $=100 \mathrm{~Hz}$,

- $\quad$ Six images shown (60 degrees)

- $\quad$ Amplitude $=1.2 \mathrm{~mm}$

- 54 black dots

- 36 images $=10$ degrees phase lag 


\section{D motion tracking}

point detection

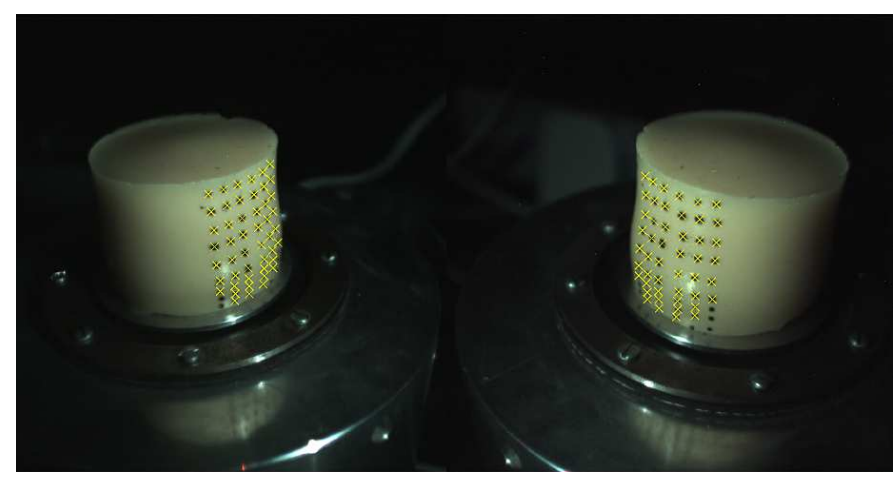

A Single Tracked Dot From the Surface of the Silicon Phantom

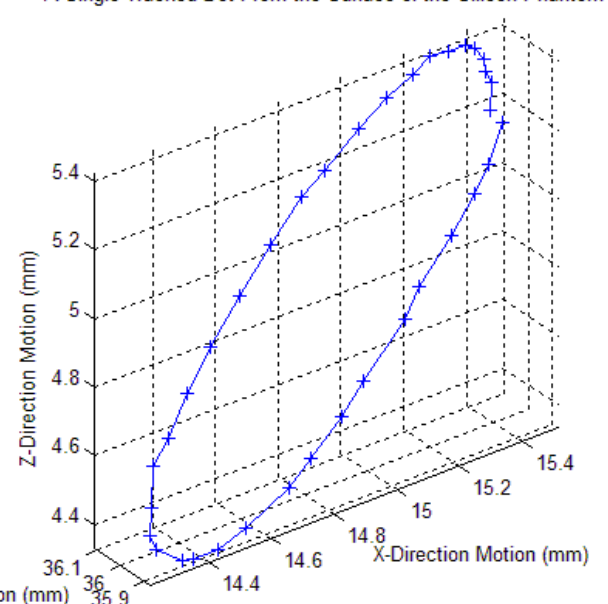

Y-Direction Motion (mm)

- Therefore captured images allowed successful tracking of the dots applied to the surface of the phantom surface reconstruction (snap shot during actuator cycle)
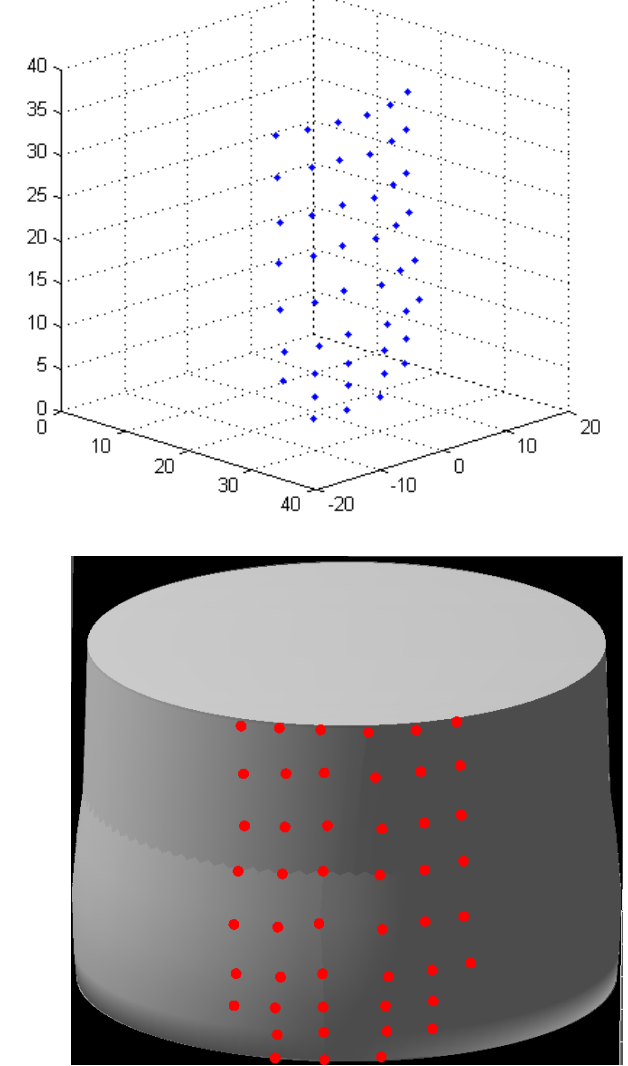

Virtual silicon phantom constructed by symmetry - agrees with visual images throughout actuation (further validation) 


\section{Further results}

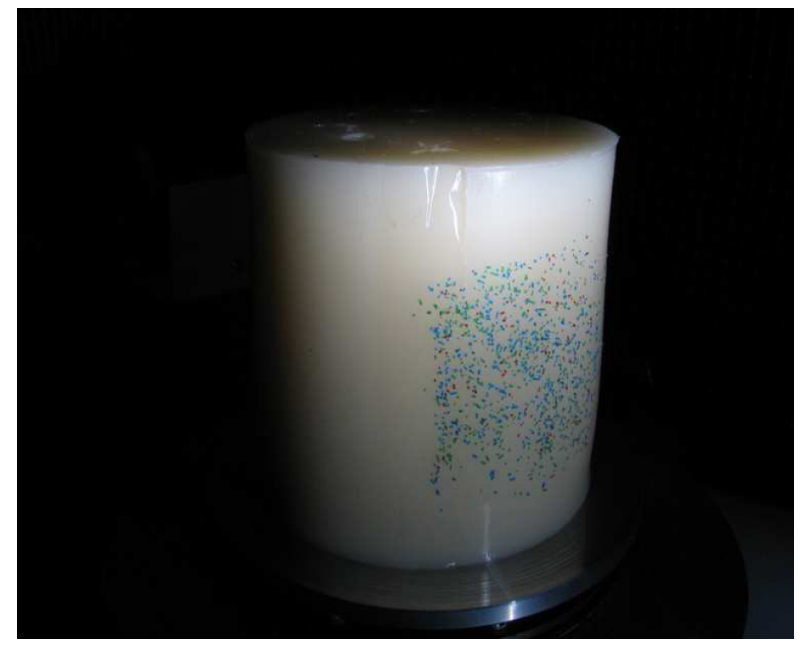

- $\quad \sim 750$ coloured fiducial marks

- 100 red, 300 blue, 350 green

- Frequency $=50 \mathrm{~Hz}, 1 \mathrm{~mm}$ peak to peak

- 20 images (18 degrees of phase)

- $\quad 90 \%$ of fiducial marks tracked successfully by point tracking method (see paper)

- Based on calibration accuracy, points are tracked within $1-2 \%$ of the magnitude of the silicon response $(<0.1 \mathrm{~mm})$
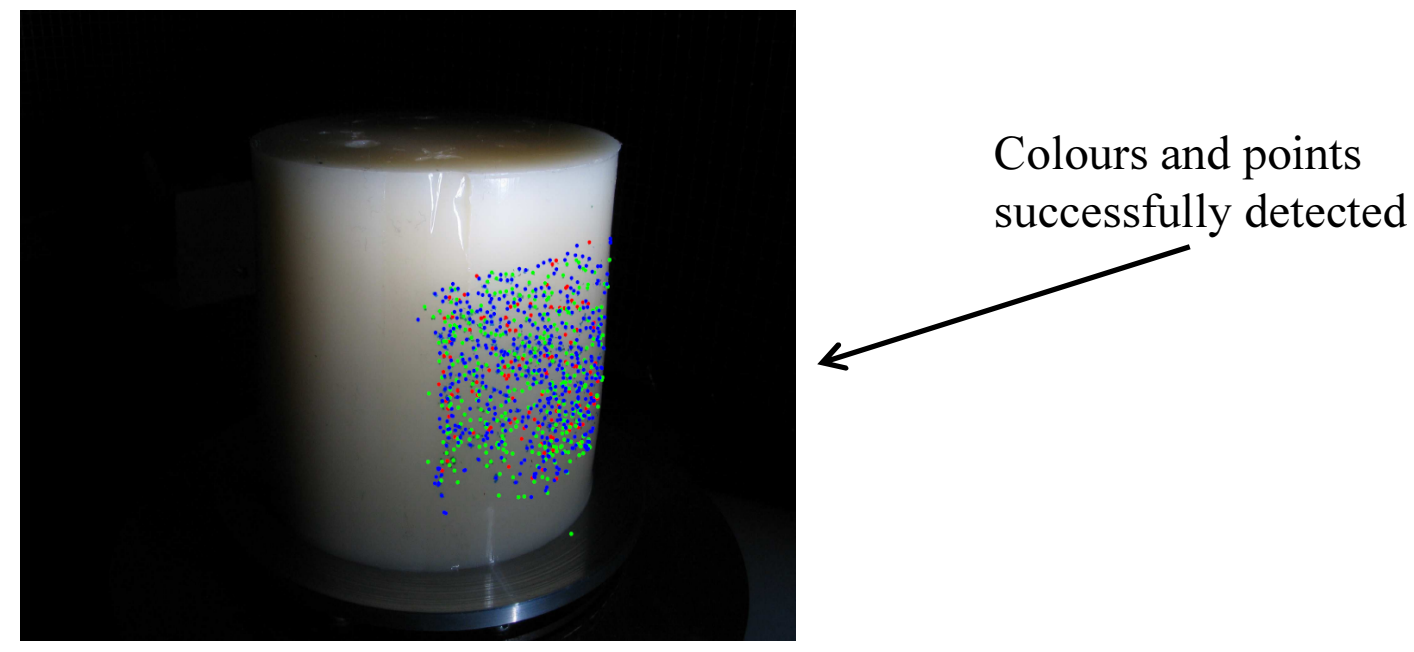

Colours and points successfully detected

\section{Example of points tracked}

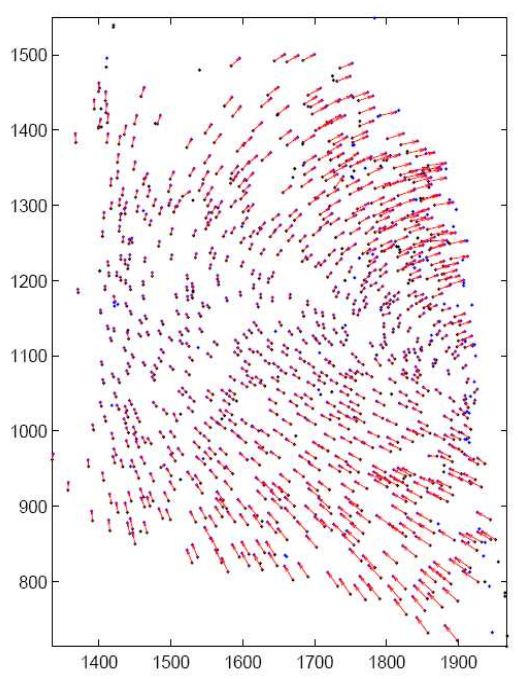

(a) whole set

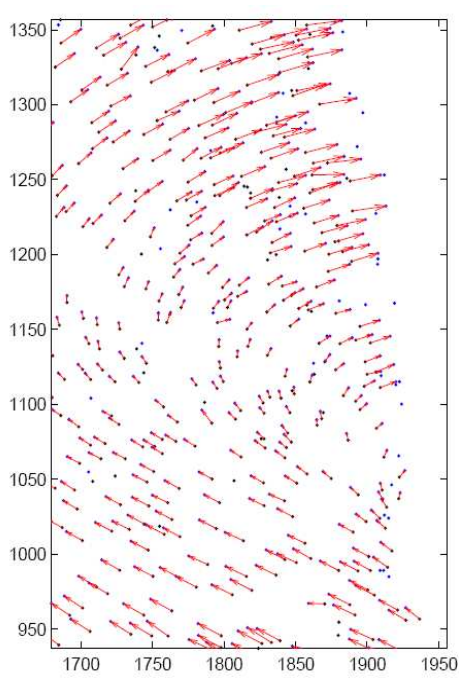

(b) Subset (zoomed in) 


\section{Conclusions}

- “Off the shelf" CMOS sensors + strobe $=$ low cost DIET imaging system

- Image capture system successfully tested from 50-100 Hz

- User-specified triggering times accurately realized within $1.4 \%$

- Captured images allowed accurate point and colour detection

- Accurate surface motion tracking at a high image resolution of 1280x1024

- $\quad$ Total capture time $=\sim 6$ minutes

- Refining of Ethernet protocols and custom design system might reduce to 20-90 seconds

- Some limitations found for current actuator (e.g. 95-100 Hz)

(to date has not shown to effect DIET system)

- Future Work:

- Replacement of the dSpace ${ }^{\mathrm{TM}}$ module with a self contained microcontroller

- Implementation of an auto focus system for the camera

- More cameras $\rightarrow$ Realistic Breast phantoms $\rightarrow$ Clinical trials 


\section{Acknowledgements}

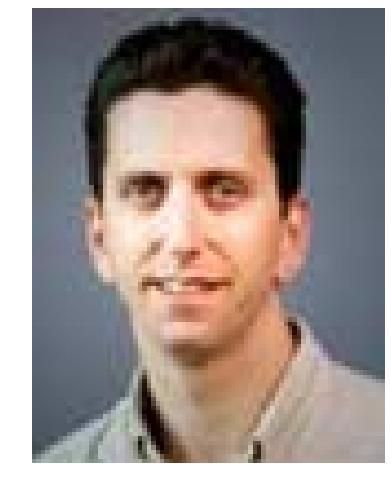

Prof. Geoff Chase

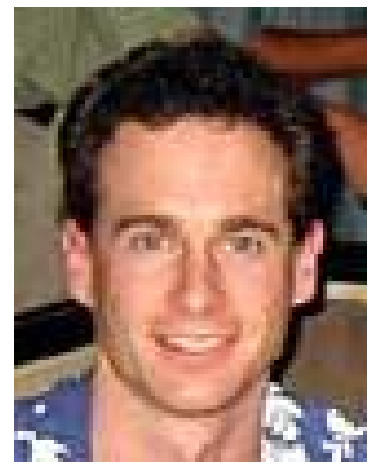

Dr. Chris Hann

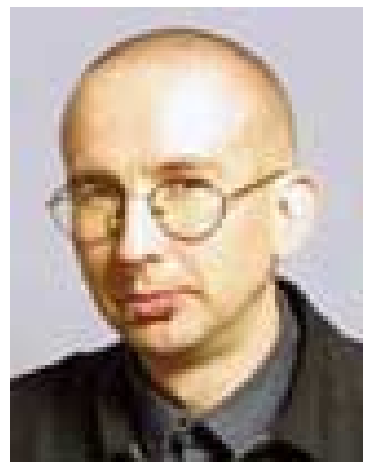

Rodney Elliot

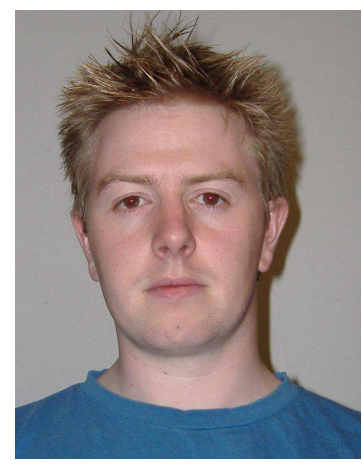

Crispen Berg

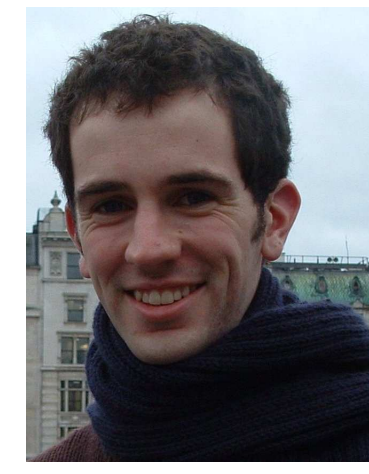

Richard Brown 